HUNGARIAN AGRICULTURAL ENGINEERING

$N^{\circ} 26 / 2014$ 29-31

Published online: http://hae-journals.org/

HU ISSN 0864-7410 (Print) / HU ISSN 2415-9751(Online)

DOI: 10.17676/HAE.2014.26.29
PERIODICAL OF THE COMITTEE OF AGRICULTURAL AND BIOSYSTEM ENGINEERING OF THE HUNGARIAN ACADEMY OF SCIENCES and SZENT ISTVÁN UNIVERSITY

Faculty of Mechanical Engineering

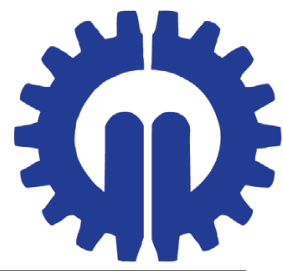

\title{
APPLE CLASSIFICATION WITH SHAPE DESCRIPTION USING 3D IMAGING TECHNOLOGIES
}

\author{
Author(s): \\ A. Lágymányosi, I. Szabó
}

\author{
Affiliation: \\ Faculty of Mechanical Engineering, Szent István University, Páter K. u. 1., Gödöllö, H-2103, Hungary \\ Email address: \\ lagymanyosi.attila@gek.szie.hu, szabo.istvan@gek.szie.hu
}

\begin{abstract}
In agricultural crop classification color and shape are the mostly investigated characteristics. In most cases classification is traditionally carried out by people using simple visualization or by automated image processing. The aim of the image processing analysis, is to search for specific shape properties of crop class, or give a description of the specific geometry of the total crop. The applied image resolution is critical parameter regarding image processing procedures. The high-resolution image data might be significantly large and so the processing usually slow, while on the other hand the essential shape characteristics may be lost due to the few pixels in the case of low resolution. In the imaging studies of shape characteristics fundamentally two main lines are being distinguished. One is the conventional twodimensional imaging, and the other is the image analyses applied on $3 \mathrm{D}$ images. In this article a $3 \mathrm{D}$ image based evaluation technique is presented which provides an additional method to apple grade classifications. With this method the conventional identification used to classify apples can be extended and so the accuracy can be improved.
\end{abstract}

\section{Keywords}

3D-imaging, image processing, shape feature

\section{Introduction}

There are several different features need to be defined together in order to precisely describe an apple. These main characteristics are colour, smell, flavour and shape. Traditionally, to assess these features the manual examination by using human sensory organs, such as vision, scent and taste, is inevitable. To define shape and colour the vision of the inspector is needed.

When machines are used for identification of the above mentioned characteristics devices with various operational principles are needed [3]. For example, in case of flavour the so called artificial tongue, while to recognize shape and colour artificial vision or machine vision is the appropriate solution [1]. The applied image processing procedure needs entirely different principles for colour and shape description.

Some of the shape characteristics of an apple can be derived from a conventional two-dimensional image [4]. In this case, the information is provided by the shape of the apple's projection.
The inspector, however, evaluates the fruit in three-dimension as default. Therefore, the person also sees the shape of those parts which remain hidden on a projected image. So as to describe an apple's characteristic shape in two dimensions it is sliced lengthwise and width wise resulting in longitudinal and cross section.

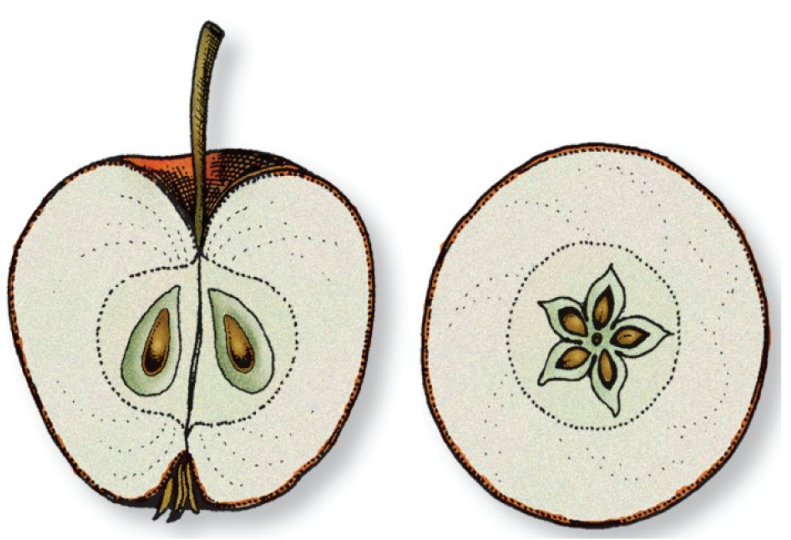

Figure 1. The apple section planes

After the fruit has been sliced sections can be well defined by means of two-dimensional image acquisition methods. This way the features of core can also be assessed which, in this particular case, is another important piece of information. Nevertheless, it is a destructive method in which the value of the fruit will finally be lost.

In classification for quality the core parameters are usually not relevant. In this case, the main classification features are shape, colour and size which are external characteristics [2]. The shape characteristics are specific to a variety and changes (possible deformations) are used as basic quality classification factor. This is underpinned by the fact that specific shape dos not necessarily define a variety but a variety has specific shape characteristics.

By using three-dimensional imaging the 3D image of the apple can be acquired. Compared to a two-dimensional image this contains numerous pieces of information concerning shape [6]. The information, however, remains hidden within the data. Dataset can be larger with orders than in case of a simple twodimensional image. So the hidden information within a threedimensional image can provide with many additional shape attributes but these can only be derived from the descriptive dataset with an appropriately chosen mathematical model. At 
present, the most limiting factor detaining the spread and use of 3D technologies is the difficulties with handling such large volume of data. Therefore, the general aim of the researchers, working on the area of $3 \mathrm{D}$ imaging, is to search and develop such algorithms by which data volume and processing time be reduced significantly [5]. There are no universal, generally applicable image processing methods! With regard to this, the ultimate aim is to always identify and develop a problem-specifically designed and tuned algorithm. In case of the 3D-image-based-appleclassification, the aim has been the same.

\section{Applied system elements}

In order to exclude the measuring errors originated in the operational principle 3D scanner two 3D scanners, with two operational principles, were used.

\section{a./ A laser scanning:}

3D laser scanner of the type Zscanner 700 was used. The main technical parameters were: sampling rate 18000 sample / sec., 2 built in cameras, improved resolution of $0,1 \mathrm{~mm}$, maximal accuracy of $\mathrm{XY}$ positioning is $50 \mu \mathrm{m}$ if the investigated volume is $100 \mathrm{~mm} \times 100 \mathrm{~mm}$.

\section{b./ Procedure based on a projected contrast grid (dark and bright bands):}

Breuckmann optoTOP -HE 1097 where the Sensor Principle of operation Miniaturised Projection Technique with Light source $100 \mathrm{~W}$ halogen, Imaging High resolution digital camera Digitizing 1384 x 1036 pixels, Operating distance from approx. $50 \mathrm{~mm}$, Min. depth resolution $2 \mu \mathrm{m}$, Acquisition time $<1 \mathrm{~s}$.

For 3D image acquisition to evaluate an apple the original software of scanners were used.

Files were saved with *.stl extension. For mathematical transformation Matlab and MS Excel software were used.

\section{Applied methode}

According to the hypothesis a given apple variety is usually classified for quality based on primarily size and then on deviation from the ideal shape. By considering the size based classification solved the shape characteristics remain in the centre of interest.

An ideal apple is considered as symmetric on the axis that connects the stem and the calyx. Parameterizing the deviation from the ideal the increase of deviation can be defined as quality reduction. By having an unambiguously quantified value which expresses the deviation of an ideal apple an exact classification into quality classes will be feasible.

The deviation can be measured in several ways. From these methods the one, which can squarely described, repeated and validated must be selected. Furthermore, the selected method should use simple calculation mechanism on preferably few input data.

The above described criteria can only be fulfilled if a welldefined reference system can also be provided. For example, the lengthwise section of an apple will obviously depend on the direction of slicing even if it is absolutely perpendicular to the width wise axis.

The fundaments of the new method, developed by authors are the followings:

- ensuring standard resolution in case of all measurement,

- transformation to a uniform orientation system,

- easily calculated and validated quality parameters.

Images generated by $3 \mathrm{D}$ scanners contain measuring points in altering densities on the object's surface. The alteration is caused by the orography of surface. The contiguous and ruption-less surface is usually described by triangulation method and with the normal vector of the surface elements. Drawback of this technique is that during generating a section a surface where no measured data available can be sliced.
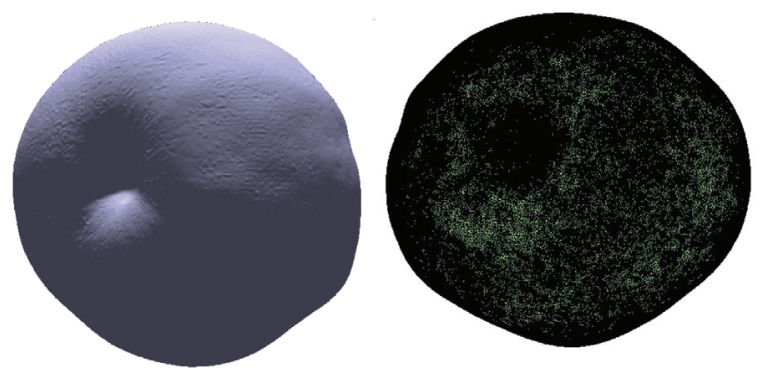

Figure 2. An apple with original $*$.stl and with variable from matrix

\section{Ensuring standard resolution}

In order to ensure same number of points that form an object a predefined (100x100x100) matrix was created uploaded with 0 values. After this, the original 3D dataset's minimum coordinates were translated to the origin. Then, all surface elements were assigned to each cell of the 0 matrix depending on the distance from the origin. In this selected cell the value was changed to 1 . This was a matrix was created where all characteristic positions of surface are 1 and every other position is 0 .

All surface elements' position was defined with closed surface with $1 \%$ accuracy at standard resolution.

This transformation resulted in (depending on the size and shape of the apple) $1 / 8$ to $1 / 10$ less surface elements than the original scanned image.

\section{Change to a uniform orientation system}

During the measurement the origin of the coordinate system and the lengthwise direction of apple depend on which scanner was used and what was the starting position of apple at the beginning of scan. The logging of minimal values was done during ensuring the standard resolution. Nevertheless, due to the differently positioned apples the translation solely does not ensure uniform reference system.

As an origin of the uniform orientation system the apples' centre of gravity was chosen. In order to eliminate the effect of random rotation among the rotation axes along the centre of gravity of an apple, considered as homogenous body, the so called inertial main axes were selected. These axes are perpendicular to each other, pair wise.

Like this, a reference system was created where all apples' longitudinal axis overlaps with one of the reference system's main axes and the origin is situated in all apples' centre of gravity.

The procedure was carried out by with the following mathematical apparatus:

If the $\mathrm{x}, \mathrm{y}, \mathrm{z}$ variables represents the apple surface points.

Js the tensor of the moment of inertial to mass centre

$$
J_{S}=\left[\begin{array}{ccc}
\sum y^{2}+z^{2} & -\sum x * y & -\sum x * z \\
-\sum x * y & \sum x^{2}+z^{2} & -\sum y * z \\
-\sum x * z & -\sum y * z & \sum x^{2}+y^{2}
\end{array}\right]
$$


Js $\_$the tensor of the primary axis, where the $\lambda 1, \lambda 2, \lambda 3$ the $\mathrm{J}_{\mathrm{s}}$ eigenvalues

$$
J_{S_{-} p}=\left[\begin{array}{ccc}
\lambda_{1} & 0 & 0 \\
0 & \lambda_{2} & 0 \\
0 & 0 & \lambda_{3}
\end{array}\right]
$$

With the $\mathrm{J}_{\mathrm{s} \_\mathrm{p}}$ create the $\mathrm{Q}$ matrix where the column represents the $\lambda 1 \ldots . . \lambda 3$ eigenvectors

$$
Q=\left[\begin{array}{lll}
\lambda_{1 x} & \lambda_{2 x} & \lambda_{3 x} \\
\lambda_{1 y} & \lambda_{2 y} & \lambda_{3 y} \\
\lambda_{1 z} & \lambda_{2 z} & \lambda_{3 z}
\end{array}\right]
$$

Finally to the coordinate transformation was used the next equation

$$
\left[\begin{array}{l}
x^{\prime} \\
y^{\prime} \\
z^{\prime}
\end{array}\right]=Q\left[\begin{array}{l}
x \\
y \\
z
\end{array}\right]
$$

\section{The selection and creation of the quality parameters}

By using the standard resolution and uniform direction of matrixes several attributes were examined. These were the comparison of torque on the selected main axes, the torque ratios on the main axes of a particular apple's and the distance between the origin and main axes' points intersecting the surface.

According to authors' experience the deviation from an eyeappeal, variety specific shaped apple are basically not the knobs on the surface but distortions, assumingly originated in development disorder. These distortions occurred near the stem and the calyx in various levels.

That is why the selected parameter, primarily due to the simple and quick calculation method, is the distance between the stem and/or calyx and the nearest main axis.

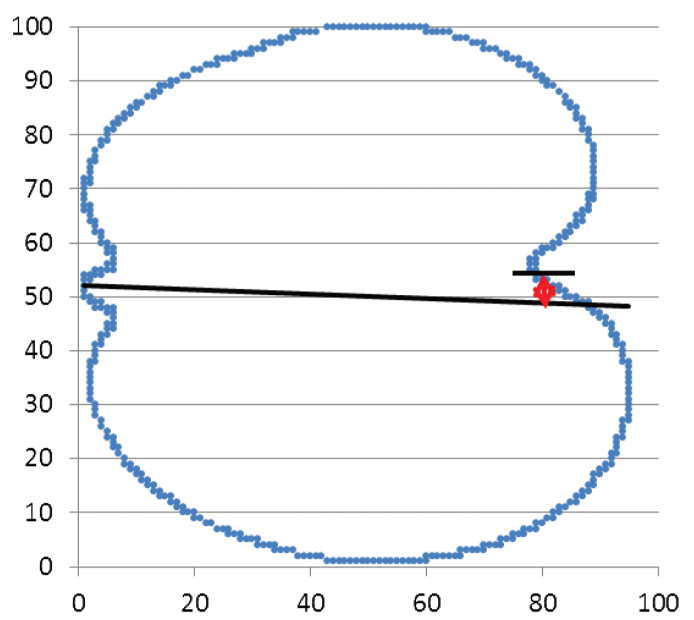

Figure 3. 2D slice with the difference from primary axis

\section{The results}

Authors have developed a method that creates a uniform reference system for evaluating the shape attributes of the apple. It is capable of treating the characteristic dataset uniformly, independently from size and orientation. Out of several shape features on have been selected that can be used to describe the apple distortion's level (deviation from ideal) by using a simple value, or value pair.

By completing the automatic classification of apples with this characteristic the quality of classification can be further increased.

\section{References}

[1] Bense L, Nagy I. Robotok a gyümölcsösben. Agrofórum, 2013. 03: 146-149. ISSN 1788-5884

[2] Hajagos A, et al The effect of rootstocks on development of fruit quality parameters of some sweet cherry (Prunus avium L.) cultivars, 'Regina' and 'Kordia', during the ripening process. 2012, Acta Universitatis Sapientiae, Agriculture and Enviroment, 4, pp. 59-70.

[3] Kátai L, et al 3D Scanning and Computer Analysis of Morphological Aspects for Agricultural Applications. In: Hungarian Agricultural Engineering, 23/2011 December p.105108. HU ISSN 0864-7410

[4] Blahunka Z. Faust D, Bártfai Z, Lefánti R. Synergy of optical insect counter and mobile robot, Synergy in The Technical Development of Agriculture and Food Industry 2011, Gödöllö, ISBN:978-963-269-250-0

[5] Kátai L, Szabó I. Digital Image Processing for Qualifying Chopped Plant Bulks.

In: Hungarian Agricultural Engineering, 1997. 10. p.35-36.

[6] Molto, E. et al An artificial vision system for fruit quality assessment Conference of European Agricultural Engineering, Madrid 1996, 96F-078 